\title{
Las estrategias de enseñanza y los estilos de aprendizaje una aproximación al caso de la licenciatura en educación de la Universidad de Cartagena (Colombia)
}

\author{
Ronald M. Marsiglia-Fuentes*, Jorge Llamas-Chávez y Edilbert Torregroza-Fuentes \\ Universidad de Cartagena, Grupo de Investigación CTS, Programa de Licenciatura en Educación con énfasis en \\ Ciencias Sociales y Ambientales, Cartagena de Indias - Colombia. (e-mail: rmarsigliaf@unicartagena.edu.co; \\ jllamasc@unicartagena.edu.co, etorregrozaf@unicartagena.edu.co)
}

* Autor a quien debe ser dirigida la correspondencia

Recibido Jul. 5, 2019; Aceptado Ago. 27, 2019; Versión final Sep. 15, 2019, Publicado Feb. 2020

\begin{abstract}
Resumen
El presente trabajo tiene como objetivo caracterizar las preferencias perceptivas y los estilos de enseñanza de mayor aceptación por parte de los estudiantes de primer semestre del programa de licenciatura en educación de la Universidad de Cartagena, en Colombia. Esos aspectos fueron indagados mediante la aplicación de la prueba de VARK (visual, funcional, lectora, quinestésica), un cuestionario de estilos de enseñanza preferido. El mayor porcentaje de los estudiantes tienen preferencia por estilo de aprendizaje lector (31.7\%) y el menor porcentaje por el estilo visual (17.1\%). El estilo de enseñanza de mayor agrado es el funcional con un $46.4 \%$, lo cual indica un mayor interés en aspectos realistas, concretos y prácticos. La información obtenida posibilitó proponer estrategias de enseñanza acorde con la preferencia perceptiva y el modo de enseñanza de mayor aceptación.
\end{abstract}

Palabras clave: estrategias de enseñanza; preferencias perceptivas; estilos de aprendizaje, VARK.

\section{Teaching strategies and learning styles an approach to the case of the degree in education of the University of Cartagena (Colombia)}

\begin{abstract}
The objective of the present work is to characterize the perceptual preferences and teaching styles of greater acceptance by freshmen students of the Degree in Education program of the University of Cartagena, in Colombia, during their first semester. These aspects were investigated through the application of the VARK test (visual, aural, read/write, kinesthetic), a preferred teaching styles questionnaire. The highest percentage of students prefer reader learning style (31.7\%) and the lowest percentage prefer visual style $(17.1 \%)$. The teaching style of greater acceptance is the functional one with $46.4 \%$, which indicates a greater interest in realistic, concrete and practical aspects. The information obtained made it possible to propose teaching strategies in accordance with perceptual preference and the most accepted teaching mode.
\end{abstract}

Keywords: teaching strategies, perceptive preferences, learning styles, VARK 


\section{INTRODUCCIÓN}

Las estrategias de enseñanza constituyen un aspecto de suma importancia para el proceso educativo, desde algunas miradas se entiende como el conjunto de decisiones que toma un docente para orientar la enseñanza con el fin de promover el aprendizaje de sus alumnos (Anijovich y Mora, 2009). De la misma manera, se asocia al como enseñar un determinado contenido disciplinar, teniendo en cuenta la realidad perceptiva y las motivaciones de los estudiantes para que comprendan con buena disposición el sentido del conocimiento que se desea compartir. Es precisamente, esas decisiones y comprensiones las que motivan a considerar como esencial el conocer los estilos de aprendizaje de los estudiantes a partir de sus preferencias perceptivas como un punto de partida desde el cual plantear adecuadas estrategias de enseñanza tanto individualmente como en el sentido grupal de un proceso que se entiende integral. Lo anterior toma mayor fuerza cuando existe consenso en considerar la enseñanza como proceso que adquiere sentido cuando impacta el aprendizaje, por lo que ambos procesos la enseñanza y el aprendizaje están tan estrechamente relacionados requiriéndose una aproximación integral e integrada de los mismos (Chiang et al., 2016).

De igual manera, y tal como lo afirmara Camilloni, para un docente no es solo importante considerar los contenidos temáticos de una disciplina sino también la manera más conveniente de trabajar esos contenidos con los estudiantes; por tanto, las estrategias didácticas junto con las temáticas son aspectos que se deben abordar de una forma articulada (Camilloni et al., 1998). Algunos autores han abordado la conceptualización sobre los estilos de enseñanza asumiéndolos en términos de comportamientos que caracterizan la enseñanza del docente. En ese orden de ideas los estilos de enseñanza serían el resultado "del cruce de los valores propios del docente con su significación de la enseñanza, así como del contexto socioeducativo-cultural donde desarrollan su docencia" (Renés et al., 2013). De tal manera que una determinada forma de enseñar estaría en concordancia con los saberes, valores y actitudes que ese docente intenta compartir.

En ese mismo sentido, diversos trabajos han señalado que los estilos de enseñanza implican categorías de comportamiento que favorecen determinados estilos de aprendizaje (Martínez-Geijo, 2009). Siendo estos estilos de gran importancia e interés en los procesos de conocimiento de los grupos de cara a asegurar un adecuado proceso educativo. Existen además una gran cantidad de cuestionarios para determinar estilos de aprendizaje considerando diversos aspectos o prioridades entre ellos el VARK que enfatiza en los canales de percepción de la información (Diago-Egaña et al., 2018), considerándose que el VARK uno de los cuestionarios de mayor presencia en diferentes motores de búsqueda y de los que mejor encaja con la semántica original del término Estilos de Aprendizaje.

Ahora bien, los estilos de enseñanza se asocian con la manera como se lleva a cabo la clase por parte del profesor, esto refiere a la forma como este organiza y elabora el plan de curso, la manera de aplicar el método y como se relaciona con los estudiantes (Ferrández y Sarramona, 1987). De la misma manera hay que tener presente que no es posible trazar parcelaciones infranqueables ya sea en cuanto a estilo de aprendizaje o estilo de enseñanza, pues a la diversidad de los grupos, se añade la propia complejidad del proceso educativo donde operan contextos cambiantes que plantea el necesario empleo de enfoques variados acordes con los interese de cada caso en particular y en atención de que determinada preferencia puede variar con el tiempo y bajo circunstancias derivadas de la naturaleza del tema o núcleo problémico que se desarrolle en la clase.

Se infiere entonces que los docentes deben esforzarse por tener información relevante acerca de las características de sus estudiantes y la manera como estos están más dispuestos, tanto individual como grupalmente, para participar en el proceso educativo dado que son sujetos esenciales para la enseñanza y el aprendizaje como un proceso integral. De otra parte, este tipo de trabajos constituyen un aporte importante orientado a la mejora de la educación en cuanto a la práctica de aula y presenta insumos necesarios para abordar no solo aspectos de caracterización del estudiante y del grupo sino plantear acciones para el mejoramiento de la calidad de la educación y en especial los procesos de enseñanza y aprendizaje (Guerra, et al., 2016).

De acuerdo a (Camarero et al., 2000) para que el desarrollo del alumno sea integral, se deben tener en cuenta los estilos y estrategias de aprendizaje. En el primer caso, se clasifican cuatro estilos de aprendizaje según la preferencia individual del alumno para adquirir conocimiento; los cuales son 1. El estilo activo basado en experiencia directa, 2. El estilo reflexivo referente a la observación y análisis de datos, 3. El estilo teórico, basado en los procesos metodológicos y estructuración y 4 . El estilo pragmático que se basa en la experimentación y búsqueda de técnicas de aplicación, que en parte viene conformados por las vivencias individuales que traen los alumnos y que conlleva a la elección de su carrera universitaria (Gonzalez, 1986). En cuanto a las estrategias como mecanismo de construcción de conocimiento a partir de la resolución de problemas (Feuerstein, 1980; Bandura, 1982; Beltrán, 1993), se establecen cuatro fases; 1. La fase de adquisición de tipo exploratorio, 2. La fase de codificación de tipo organizacional, 3. La fase de recuperación, para generar respuestas y 4 . La fase de apoyo, que implica la autocrítica y el apoyo de la sociedad. 
El presente estudio se orienta precisamente a determinar cuáles son las preferencias en cuanto a estilos de aprendizaje predominantes en estudiantes del primer ciclo de la Licenciatura en Educación con énfasis en Ciencias Sociales y Ambientales y de igual manera, realiza un acercamiento en cuanto a estrategias de enseñanza empleadas por los docentes que son de preferencia por parte del estudiante.

\section{METODOLOGÍA}

Se plantea un estudio de tipo descriptivo, dado que se orienta a conocer los estilos de aprendizaje predominantes, así como los estilos de enseñanza alrededor de los cuales existe preferencia por parte de los estudiantes que cursan el primer ciclo de la Licenciatura en Educación con énfasis en Ciencias Sociales y Ambientales.

Grupo de estudio: El grupo de estudiantes seleccionados para el desarrollo de la valoración fueron los 30 estudiantes cursaban el primer semestre del Programa de Licenciatura en Educación con énfasis en Ciencias Sociales y Ambientales de la Universidad de Cartagena durante el año 2018. De estos 20 eran mujeres y 10 varones. Es importante señalar que inicialmente el listado estaba conformado por 33 estudiantes, pero tres (3) de ellos desertaron y no hicieron parte del proceso por lo que fueron desestimados en las ponderaciones y análisis que se derivaron.

Estilos de aprendizaje: El estudio sobre estilos de aprendizaje se realizó mediante el test de VARK propuesto por Fleming que incluyo la aplicación de un instrumento que, a manera de identificación de preferencias, posibilita la reflexión y el análisis de “¿Cómo aprendo más rápido y mejor?” y “¿En cuales condiciones?”. Este instrumento denominado VARK corresponde al acrónimo de las cuatro letras iniciales de las preferencias modales sensoriales (Visual, Aural, Read/Write, Kinesthetic por su sigla en inglés) (Visual, Auditivo, Lectura/Escritura y Kinestésico), propuesto por Neil Fleming junto con Collen Mills. Es importante señalar que el instrumento consta de 16 preguntas con cuatro posibles respuestas (Fleming, 2001).

Estilos de enseñanza: Para determinar el estilo de enseñanza de mayor agrado o preferencia por parte de los estudiantes se consideraron cuatro (4) categorías propuestas por Renés y colaboradores, que delimitan cuatro Estilos de Enseñanza: Abierto, Formal, Estructurado y Funcional (Renés et al., 2013). El Cuestionario de Estilos de Enseñanza Preferido (CEEP) por parte de los estudiantes se estructuro de 20 ítems. Adicionalmente, se incluyó un espacio para describir con sus propias palabras el tipo de estilo de enseñanza que espera de los profesores y maestros que imparten los cursos en el programa.

\section{RESULTADOS Y DISCUSIÓN}

Una vez aplicado el instrumento propuesto por Fleming (Test de VARK), referido a caracterizar los estilos de aprendizaje de los estudiantes del Programa, se encontraron los siguientes resultados individualizados y porcentuales de cada modo sensorial; los cuales se muestran en la tabla 1

Tabla 1: Relación porcentual de preferencias perceptivas de los estudiantes

\begin{tabular}{|c|c|c|c|c|c|c|}
\hline$N^{\circ}$ Listado & Género & $\%$ Visual & $\%$ Auditivo & $\%$ Lector & $\%$ kinestésico & Preferencia Sensorial \\
\hline 1 & $\mathrm{~F}$ & 13,0 & 30,4 & 34,8 & 21,7 & Lector \\
\hline 2 & $\mathrm{~F}$ & 18,8 & 6,3 & 62,5 & 12,5 & Lector \\
\hline 3 & $\mathrm{~F}$ & 10,3 & 31,0 & 31,0 & 27,6 & Auditivo-Lector \\
\hline 4 & $\mathrm{M}$ & 12,5 & 18,8 & 37,5 & 31,3 & Lector \\
\hline 5 & $\mathrm{~F}$ & 10,5 & 36,8 & 31,6 & 21,1 & Auditivo \\
\hline 6 & $\mathrm{~F}$ & 26,1 & 17,4 & 39,1 & 17,4 & Lector \\
\hline 7 & $\mathrm{M}$ & 7,1 & 28,6 & 32,1 & 32,1 & Lector-Kinestésico \\
\hline 8 & $\mathrm{M}$ & 25,0 & 12,5 & 31,3 & 31,3 & Lector-Kinestésico \\
\hline 9 & $\mathrm{M}$ & 25,0 & 21,4 & 35,7 & 17,9 & Lector \\
\hline 10 & $\mathrm{M}$ & 18,8 & 12,5 & 31,3 & 37,5 & Kinestésico \\
\hline 11 & $\mathrm{~F}$ & 6,3 & 50,0 & 18,8 & 25,0 & Auditivo \\
\hline 12 & $\mathrm{~F}$ & 33,3 & 22,2 & 22,2 & 22,2 & Visual \\
\hline 13 & $\mathrm{~F}$ & 10,5 & 36,8 & 21,1 & 31,6 & Auditivo \\
\hline 14 & $\mathrm{M}$ & 26,3 & 18,4 & 34,2 & 21,1 & Lector \\
\hline 15 & $\mathrm{~F}$ & 6,3 & 12,5 & 62,5 & 18,8 & Lector \\
\hline 16 & $\mathrm{M}$ & 14,3 & 42,9 & 21,4 & 21,4 & Auditivo \\
\hline 17 & $\mathrm{~F}$ & 12,5 & 20,8 & 41,7 & 25,0 & Lector \\
\hline
\end{tabular}


Tabla 1: (continuación)

\begin{tabular}{|c|c|c|c|c|c|c|}
\hline$N^{\circ}$ Listado & Género & $\%$ Visual & $\%$ Auditivo & $\%$ Lector & $\%$ kinestésico & Preferencia Sensorial \\
\hline 18 & $\mathrm{~F}$ & 6,3 & 43,8 & 31,3 & 18,8 & Auditivo \\
\hline 19 & $\mathrm{~F}$ & 36,0 & 32,0 & 24,0 & 8,0 & Visual \\
\hline 20 & $\mathrm{M}$ & 31,3 & 31,3 & 25,0 & 12,5 & Visual- Auditivo \\
\hline 21 & $\mathrm{~F}$ & 20,0 & 45,0 & 20,0 & 15,0 & Auditivo \\
\hline 22 & $\mathrm{~F}$ & 30,4 & 39,1 & 30,4 & 0,0 & Auditivo \\
\hline 23 & $\mathrm{M}$ & 7,7 & 23,1 & 53,8 & 15,4 & Lector \\
\hline 24 & $\mathrm{~F}$ & 25,8 & 29,0 & 19,4 & 25,8 & Auditivo \\
\hline 25 & $\mathrm{~F}$ & 12,5 & 12,5 & 37,5 & 37,5 & Lector-Kinestésico \\
\hline 26 & $\mathrm{~F}$ & 7,1 & 28,6 & 42,9 & 21,4 & Lector \\
\hline 27 & $\mathrm{~F}$ & 6,3 & 43,8 & 31,3 & 18,8 & Auditivo \\
\hline 28 & $\mathrm{M}$ & 20,0 & 33,3 & 13,3 & 33,3 & Auditivo-Kinestésico \\
\hline 29 & $\mathrm{~F}$ & 8,0 & 36,0 & 20,0 & 36,0 & Auditivo-Kinestésico \\
\hline 30 & $\mathrm{~F}$ & 0,0 & 37,5 & 37,5 & 25,0 & Auditivo-Lector \\
\hline
\end{tabular}

La relación porcentual de los participantes en el estudio considerando su género se muestra la figura 1 siendo el género femenino el de mayor participación durante el estudio de aplicación del test de VARK, con un 67\% si se compara con el 33\% de porcentaje de participación de los varones, considerando la totalidad de los participantes que constituyen el grupo de estudiantes.

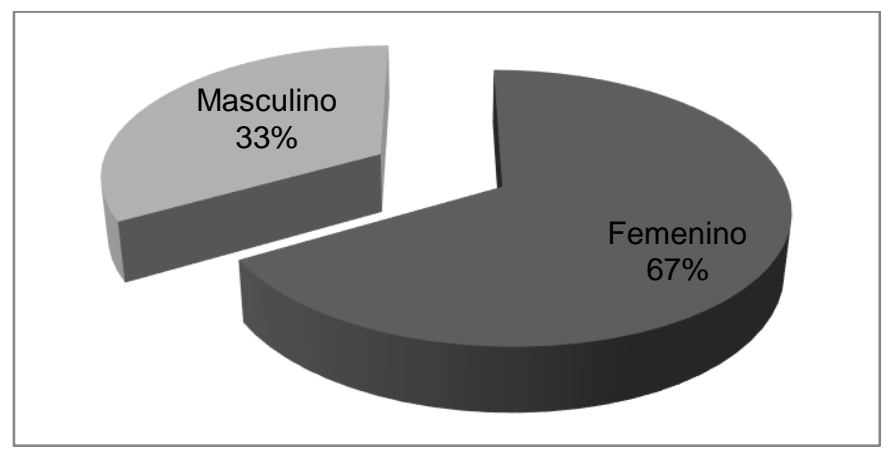

Fig. 1: Relación porcentual de los participantes según su género

Los resultados individualizados, que se representan mediante el gráfico radial de la figura 2, evidencian que a nivel de grupo de estudiantes el mayor porcentaje de los estudiantes tienen marcada preferencia por estilo de aprendizaje Lector (31.7\%) y el menor porcentaje de ellos se inclina por el estilo visual (17.1\%). La representación gráfica de esos resultados grupales, se muestran en la figura 3.

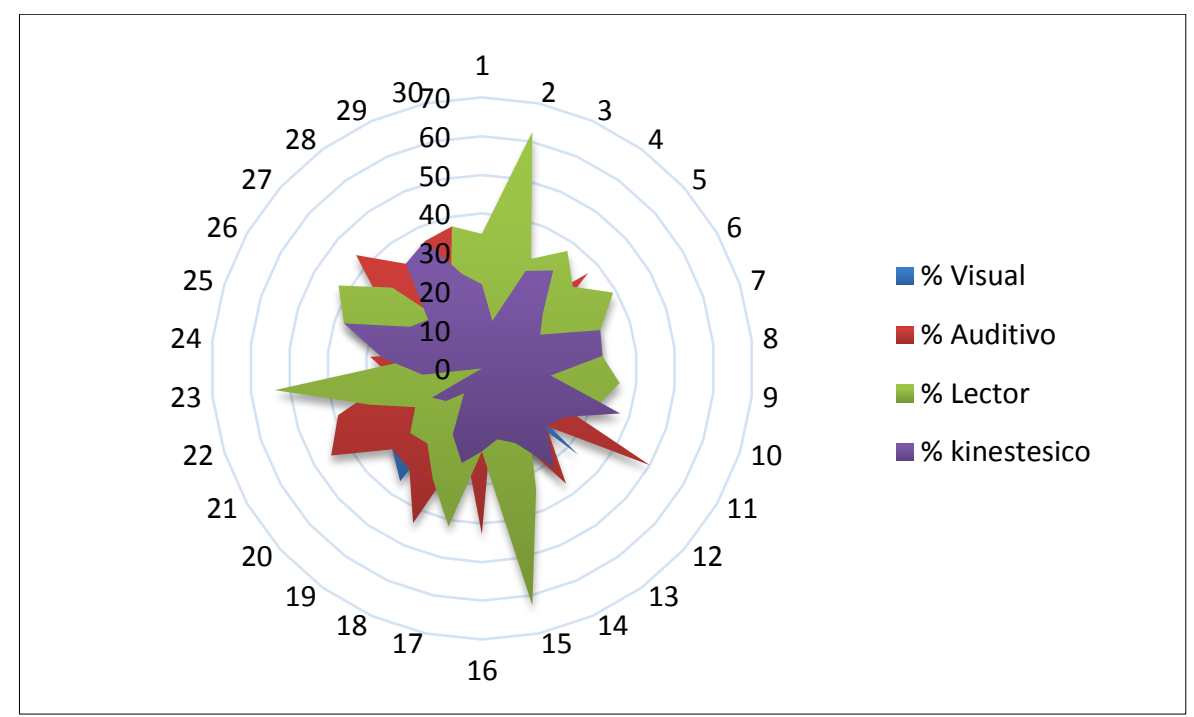

Fig. 2: Preferencia sensorial individualizada mediante Test de VARK visualizada por gráfico radial 


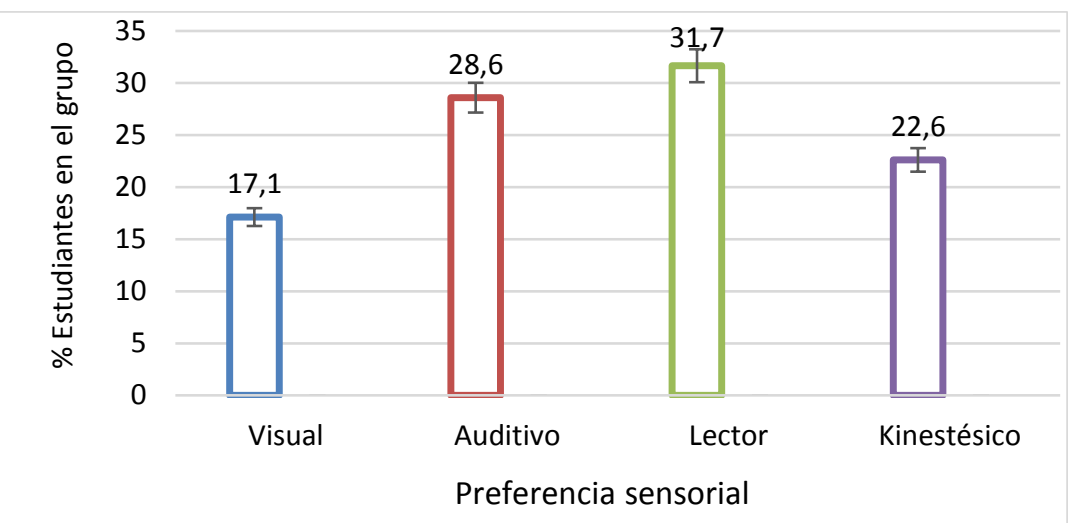

Fig. 3: Resultado grupal sobre preferencias perceptiva por parte de los estudiantes

Teniendo presente que una misma persona, atendiendo al modelo VARK aplicado, puede manifestar simultáneamente preferencia por varios estilos de aprendizaje; se muestra a continuación la diferencia por género. Encontramos que para el grupo de mujeres (femenino) el $55 \%$ y el $45 \%$ del total de las 20 estudiantes tienen preferencia por estilos de aprendizaje auditivo y lector respectivamente; mientras que a nivel de los estudiantes varones (masculino), del total de 10 que participaron en el estudio, se encontró que un $60 \%$ de ellos tienen preferencia por el estilo de aprendizaje lector y un $40 \%$ manifestó preferencia sensorial kinestésica. Sin embargo, a nivel de estilo de aprendizaje visual, se encontró que tanto varones como mujeres presentan un $10 \%$ de preferencia por este tipo de percepción. Los resultados desglosados por genero se muestran en la figura 4.

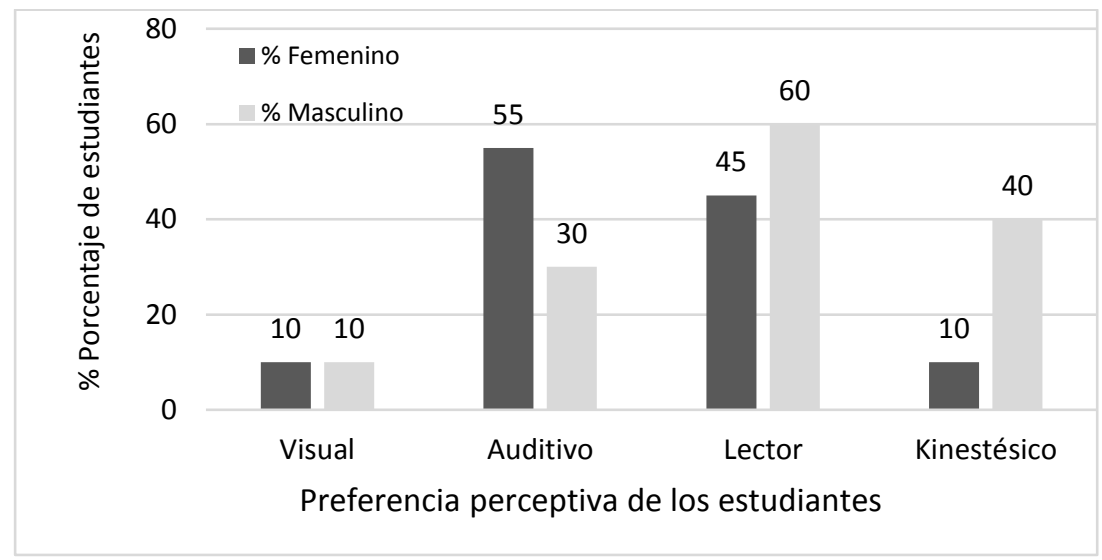

Fig. 4: Estilos de aprendizaje desglosados por género

Los resultados desglosados por genero muestran que, para el caso estudiado, tanto varones como mujeres tienen en el estilo lector una de las preferencias perceptivas de mayor porcentaje $60 \%$ y $45 \%$ respectivamente y el resultado más dispar se encontró en el estilo kinestésico al reportarse $10 \%$ para las mujeres y $40 \%$ en varones. Resulta llamativo el hallazgo que muestra para ambos segmentos un idéntico porcentaje en cuento a estilo perceptivo visual (10\%); lo cual indica que estas diferencias y similitudes no constituyen una generalización, pues otros estudios han expresado que los varones son más visuales que las mujeres (Olarte, 2017), afirmación esta última que no se evidencio en el presente caso. Los resultados en cuanto a la determinación del estilo de enseñanza de mayor agrado o preferencia por parte de los estudiantes, considerando las cuatro (4) categorías o estilos de enseñanza (abierto, formal, estructurado y funcional), se representan en la figura 5 :

El estilo de enseñanza de mayor agrado por parte de los estudiantes es el funcional ( $46.4 \%$ ), lo cual nos indica un mayor interés en llevar a la práctica lo planificado durante el proceso de enseñanza, así como partidarios del trabajo en equipo y un fuerte deseo que los docentes orienten su esfuerzo de enseñanza hacia aspectos realistas, concretos y prácticos, evitando divagar en largas explicaciones, de tal manera que exista un adecuado equilibrio entre lo teórico y lo práctico. Se destaca igualmente, que existe una menor preferencia por el estilo de enseñanza formal (3.5\%), quizás debido a lo poco flexible del mismo y al hecho que el programa de licenciatura para el caso en estudio promueve y privilegia el trabajo en equipo como una expresión importante de su modelo pedagógico, mientras que el estilo de enseñanza formal es más partidario del trabajo individual sobre el grupal. 


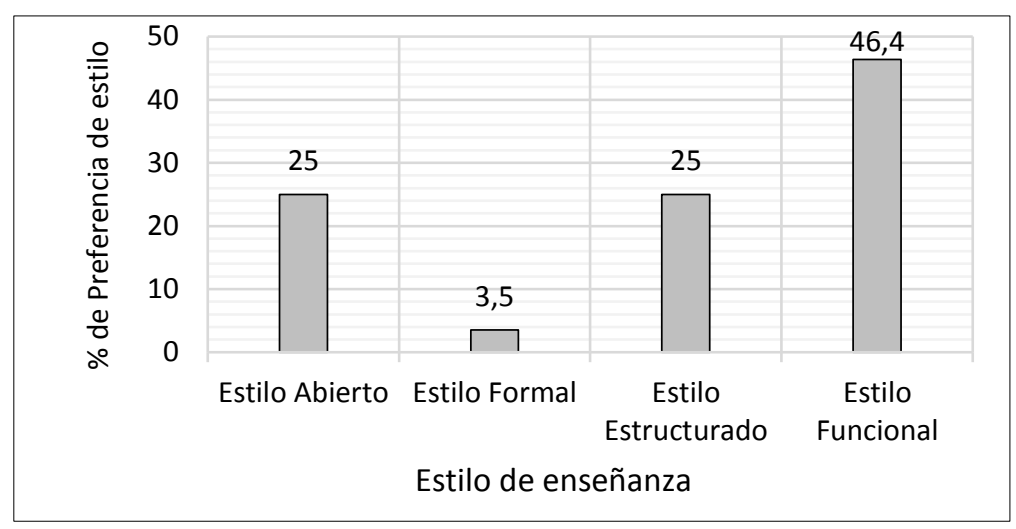

Fig. 5: Preferencia en cuanto a estilos de enseñanza

Para el caso de los estudiantes que manifiestan una preferencia perceptiva de estilo Kinestésico (22,6\%), las estrategias de enseñanza recomendables se orientan al uso de estrategias tales como: juego de roles y dramatizaciones, dinámicas grupales que requieran sentarse y pararse, el pizarrón para resolver problemas, manipulación de objetos para explicación de fenómenos, así como el uso de gestos para acompañar las instrucciones orales. Los estudiantes que manifestaron una preferencia por el estilo visual (17,1\%), las estrategias de enseñanza que se pueden recomendar implican el uso de: videos, mapas conceptuales, orientaciones por escrito, diagramas, modelos, cuadros sinópticos, animaciones computacionales, imágenes de fotografías, transparencias e ilustraciones. Aquí es importante como estrategia de enseñanza por parte de los docentes el recomendar a los estudiantes el dibujar, realizar ilustraciones para apropiar el vocabulario nuevo, así como el leer historias y visualizar los personajes y procesamiento de documentos que consisten en análisis, síntesis, deducción, inducción y comparación (Jurenka, et al., 2018; Starecek, et al.,2018).

Considerando el grupo total de estudiantes que han manifestado una mayoritaria preferencia por el estilo de aprendizaje perceptivo lecto-escritor (31,7\%), se motivan tal como lo plantea Lozano (2001) por estrategias de enseñanza que impliquen el uso de: escritos cortos, composiciones literarias, diarios, reportes, elaboración de resúmenes, reseñas y síntesis de textos, así como mediante la revisión de textos de los compañeros. De la misma manera, podríamos plantear dentro de las estrategias para la enseñanza, el uso de canales de comunicación de tipo escrito como los mensajes electrónicos, el mural de anuncios, el dialogo epistolar y la discusión temática en foros virtuales (Olarte, 2017).Los estudiantes que han manifestado una preferencia por el estilo perceptivo auditivo (28,6\%), se recomienda el desarrollo de estrategias de enseñanza que impliquen el uso de: instrucciones verbales, repetir sonidos parecidos, audios, debates, discusiones, lluvia de ideas, leer el mismo texto con distinta reflexión, así como la lectura guiada y comentada. De la misma manera, se recomienda a estos estudiantes el realizar y participar en debates, preguntarse unos a otros, escuchar grabaciones sobre temáticas de interés, realizar lecturas en voz alta, así como grabarse a sí mismos. Ahora bien, considerando que hay un grupo de estudiantes que manifiesta preferencia por los estilos de aprendizaje auditivo y lector (figura 6); a la vez que hay un fuerte interés preferencial por el estilo de enseñanza funcional, encontramos importante proponer la lectura y el debate argumentado sobre temáticas concretas de la realidad local o regional.

Lo anterior en coincidencia con lo expresado por los estudiantes al señalar "que el encuentro de clase sea dinámico, rompiendo la rutina, con ejemplos y comparaciones de la vida real....". Para el caso de los estudiantes que manifiestan una preferencia perceptiva de estilo Kinestésico $(22,6 \%)$, las estrategias de enseñanza recomendables se orientan al uso de estrategias tales como: dramatizaciones, dinámicas grupales que pueden incluir obras de teatro, actividades que requieran movimiento, el desarrollo de rondas o el pasar al frente de la clase para orientar dinámicas, manipulación de objetos para explicación de fenómenos, así como el empleo de la gesticulación para acompañar las instrucciones orales.

Los estudiantes que manifestaron una preferencia por el estilo visual $(17,1 \%)$, las estrategias de enseñanza que se pueden recomendar implican el uso de: instrucciones escritas, mapas conceptuales, diagramas, modelos, cuadros sinópticos, animaciones computacionales, videos, transparencias, fotografías e ilustraciones. Aquí es importante como estrategia de enseñanza por parte de los docentes el recomendar a los estudiantes el dibujar, el realizar ilustraciones para apropiar el vocabulario nuevo, así como el leer historias y visualizar los personajes.

\section{DISCUSIÓN FINAL}

Los resultados a nivel grupal en cuanto a preferencias de estilo de aprendizaje, que de forma mayoría se encontró para los estilos auditivo y lector, y la preferencia funcional en cuanto a la enseñanza posibilitan indicar que para el grupo la percepción acústica y visual deben ser consideradas como un aspecto importante 
en las labores de gestión y planeación del proceso educativo y en la organización del aprendizaje de los estudiantes. Siendo estos resultados también de mucho interés al momento de seleccionar las estrategias de enseñanza que, para el caso de estos hallazgos investigativos, han decantado hacia la articulación teóricopráctica, el trabajo en equipo y la contrastación con la realidad contextual dinámicas propias del estilo funcional de enseñanza. De otra parte, este tipo de estudios relacionados con el primer año universitario constituyen un tramo considerado crítico en la definición de trayectorias estudiantiles exitosas (Silva-Laya, 2011), ya que aportan elementos valiosos para el diagnóstico educativo del estudiantado a nivel grupal e individual y además ayuda a los docentes en la selección de estrategias adecuadas.

En ese sentido, al contrastar con un estudio similar realizado con estudiantes de primer año de la Universidad de Emory (USA), orientado a diagnosticar su estilo de aprendizaje mediante la prueba de VARK, encontró que los estudiantes tienen diferentes estilos de aprendizaje y esto incide en la forma como aprenden (Marcy, 2001). El estudio realizado en Emory señalo que el cuestionario VARK es una herramienta fácil de emplear y puede mejorar el aprendizaje de los estudiantes. En ese estudio ninguno de los estudiantes participantes se identificó con preferencias de aprendizaje visuales o auditivas. Se encuentra por tanto coincidencia en el hecho de no existir homogeneidad alrededor de la preferencia sensorial cuando se caracteriza este aspecto en un grupo de estudiantes. De cualquier manera, los resultados mostraron igualmente que existen preferencias perceptivas del orden Kinestésico y visual que, aunque en menor porcentaje deben ser tenidos muy de presente por parte del cuerpo docente durante la planeación de actividades, aprovechando la asignación trabajos en equipo para realizar el abordaje diferenciado de tareas o talleres que se asignen. Lo anterior implica por igual las preferencias de enseñanza en cuanto a estilo abierto, estructurado y formal, por lo que se hace necesario tener presente la diversidad de los grupos en su conjunto pensando no solo en el estilo preferencial mayoritario, sino también en los minoritarios.

El trabajo realizado nos conduce a alejarnos de la tradicional mirada centrada en el docente, para adentrarnos en la perspectiva preferencial de los estudiantes. Es importante resaltar que el rol del profesor como orientador en la promoción de los estudiantes para el aprendizaje dinámico a través de las herramientas tecnológicas disponibles, es de gran importancia, ya que motiva al estudiante a apropiar información según las preferencias y aptitudes frente a la disposición de aprendizaje (Arteaga y Fabregat, 2002) y a su contexto educativo, donde se incentive el uso de los repositorios bibliográficos para la selección de recursos que mejoren las prácticas de formación de los estudiantes (Lerís y Sein-Echaluce, 2011; Fernández, et al., 2018).

Ahora bien, resultados de otros estudios similares permiten concluir que es ventajoso adecuar las estrategias de enseñanza al estilo de aprendizaje de los estudiantes, como medio para mejorar la calidad educativa (Durán y Costaguta, 2008), lo que nos muestra que aspectos como el abordado en el presente estudio aportan elementos valiosos para el fortalecimiento del acto pedagógico. De la misma manera, otros resultados revelan que los estilos de aprendizaje y enseñanza no son excluyentes entre sí (Moreno, et al., 2014). Encontrando además Moreno y colaboradores que para el caso de los estudiantes de licenciatura en educación básica de una facultad de estudios a distancia estos tienden hacia el estilo de aprendizaje visual y kinestésico, resultado que se aleja de lo encontrado para el grupo de estudiantes de la Licenciatura en Educación con énfasis en Ciencias Sociales y Ambientales, donde los estilos lector y auditivo fueron los de mayor tendencia.

\section{CONCLUSIONES}

Es importante tener información individual y grupal de los estudiantes para poder considerar algunas estrategias, que permitan facilitar el proceso enseñanza y aprendizaje teniendo presente la orientación de contenidos temáticos del orden disciplinar y el estilo de aprendizaje de los estudiantes que entre otros aspectos caractericen sus potencialidades y preferencias. A través del test de VARK se logró recolectar información relevante sobre las preferencias de estilo de aprendizaje, posibilitando una mejor orientación e integralidad de los procesos formativos de los futuros educadores. El estudio realizado para el grupo seleccionado mostro que los estilos auditivos (28.6\%) y lector (31.7\%) tienen el mayor porcentaje de preferencia en cuento a estilo de aprendizaje acorde con el modelo VARK; mientras que el estilo funcional (46.4\%) constituye el modo mayoritario en cuanto preferencia de estilo de enseñanza.

\section{AGRADECIMIENTOS}

Los autores agradecen el apoyo recibido en cuanto a recursos del Plan de Fortalecimiento para grupos de Investigación por parte de la Vicerrectoría de Investigaciones de la Universidad de Cartagena otorgados mediante Resolución 00334 de 2018.

\section{REFERENCIAS}

Anijovich, R. y S. Mora, Estrategias de Enseñanza: Otra Mirada al Quehacer en el Aula. Aique Grupo Editor. $1^{\text {a }}$ Ed. Buenos Aires. Argentina. (2009). 
Arteaga, C. y R. Fabregat, Integración del Aprendizaje Individual y del Colaborativo en un Sistema Hipermedia Adaptativo. DOI://lsm.dei.uc.pt/ribie/docfiles/txt2003729191353paper170. (2002).

Bandura, A., Teoría del Aprendizaje Social en un Sistema Hipermedia Adaptativo. JENUI, 2(2), 107 -114. Madrid: EspañaCalpe (1982).

Beltrán J., Procesos, Estrategias y Técnicas de Aprendizaje. $1^{\text {a }}$ Ed. Síntesis Madrid (1993).

Camarero, F. J., M. del Buey, F. de Asís y F.J. Herrero, Styles and learning Strategies in University Students. Psicothema, 12(4), 615-622 (2000).

Camilloni A., S. Celman, E. Litwin y M. Palou de Maté, La evaluación de los Aprendizajes en el debate didáctico contemporáneo. Paidós Editorial. Primera Edición. ISBN 950-12-2129-6. Buenos Aires. Argentina. (1998).

Chiang M.T., C. Díaz y P. Arriagada, ¿Teaching and Learning Styles: How to Dialogue in Practice?, Journal of Learning Styles Vol.9 N¹7 (2016).

Chiang, M.T., C. Díaz, A. Rivas y P. Martínez-Geijo, Validación del Cuestionario Estilos de Enseñanza (CEE). Un Instrumento Para el Docente de Educación Superior. Revista Estilos de Aprendizaje. 12, (11) (2013).

Diago, M.L., M.J. Cuetos y P. González, Análisis de las herramientas de medición de los Estilos de Aprendizaje. DOI: 10.4438/1988-592X-RE-2017-381-382 Revista de Educación, 381, 95-131 (2018).

Durán, E. y R. Costaguta, Experiencia de Enseñanza Adaptada al Estilo de Aprendizaje de los Estudiantes en un Curso de Simulación. doi.org/10.4067/S0718-50062008000100004. Formación Universitaria, 1(1), 19-28 (2008).

Fernández, O.G., J.B. Vázquez, y E.O. Cornejo, Promoción de la Autogestión a través de Objetos de Aprendizaje Adaptativos en Alumnos de Educación Superior. Edutec. Revista Electrónica de Tecnología Educativa, (63), 15-28 (2018).

Ferrandez, A. y J. Sarramona, Didáctica y Tecnología de la Educación. Diccionario de Ciencias de la Educación. Madrid: Anaya. (1987).

Feuerstein, R., Y. Rand y M.D. Hoffman, Instrumental Enriche Men. An Intervention Program for Cognitive Modificability. Baltimore: University Press (1980).

Fleming, N., Teaching and Learning Styles: VARK Strategies, Editorial Christchurch, Nueva Zelanda. (2001).

González-Tirados, R.M., Estudio de la fiabilidad y validez del inventario de estilos de aprendizaje. Bordón, 262 (3-4), 277292 (1986).

Guerra Pulido E., O. Pérez y P. Martínez-Geijo, Estilos de Enseñanza y Rendimiento Académico. Revista de Estilos de Aprendizaje. Fall Edition. Vol. 9 No. 18 (2016).

Jurenka, R., A. Starecek, N. Vranakova y D. Caganova, The learning Styles of the Generation Group Z and their Influence on Learning Results in the Learning Process. Paper presented at the ICETA 2018 - 16th IEEE. International Conference on Emerging eLearning Technologies and Applications, Proceedings, 251-260. DOI:10.1109/ICETA.2018.8572186 (2018).

Lerís, D., y M.L. Sein- Echaluce, The Personalization of Learning: A Goal of Educational Paradigm Focused on Learning. Arbor,187(Extra_3), 123-134 (2011).

Lozano, A., Estilos de Enseñanza y Aprendizaje. Un Panorama de la Estilística Educativa. México, $1^{\text {a }}$ Ed. Trillas: ITESM (2001).

Martínez-Geijo, P., Estilos de enseñanza: conceptualización e investigación, Revista Estilos de Aprendizaje, N.ำ 3, Vol. 2. (2009).

Olarte Ramos, C.A., Masculinidad y afectividad en el espacio público: La percepción de los varones ante las prescripciones sociales de género. Análisis en una Universidad de Tabasco. Universidad Veracruzana. Xalapa de Enríquez. México. 273p (2017).

Mercy, V., Adult Learning Styles: How the VARK Learning Style Inventory Can Be Used to Improve Student Learning. Perspective on Physician Assistant Education, Vol. 12, No 2, spring, pp 117-120 (2001)

Moreno, C., Y. Molina y J. Chacón, Impacto del estilo pedagógico integrador en los estudiantes de licenciatura en educación básica de la facultad de estudios a distancia. doi: 10.4067/S0718-50062014000600005. Formación Universitaria. Volumen 7(6), 37-44 (2014).

Renés, P., L. Echeverry y otros tres autores, Teaching styles: A step Forward in its Conceptualization and Diagnosis. Magazine of learning styles, ISSN-e 2332-8533, Vol. 6, №. 11, 2013, págs. 4-18 (2013).

Silva-Laya, M., El primer año universitario: Un tramo crítico para el éxito académico, Perfiles educativos, 33, $102-114$. (2011).

Starecek, A., M. Bednarikova, y K. Koltnerova, The level of Cognitive Abilities of Generational Group y and Educational Process. ICETA 2018 - 16th IEEE International Conference on Emerging eLearning Technologies and Applications, Proceedings, pp. 511-518 (2018). 